\title{
Final Design and Current Status of the Mu2e Crystal Calorimeter
}

\author{
N. Atanov, V. Baranov, J. Budagov, Y. I. Davydov, V. Glagolev, V. Tereshchenko, Z. \\ Usubov and I. I. Vasilyev \\ Joint Institute for Nuclear Research, Dubna, Russia \\ F. Cervelli, S. Di Falco, S. Donati, C. Ferrari, A. Gioiosa, V. Giusti, A. Marini, L. \\ Morescalchi*, D. Pasciuto, E. Pedreschi, F. Raffaelli, F. Spinella and A. Taffara \\ INFN - Sezione di Pisa, Pisa, Italy \\ C. Bloise, F. Colao, M. Cordelli, G. Corradi, E. Diociaiuti, R. Donghia, S. \\ Giovannella, D. Hampai, F. Happacher, M. Martini, S. Miscetti, D. Paesani, A. \\ Saputi and I. Sarra \\ Laboratori Nazionali di Frascati dell'INFN, Frascati, Italy
}

L. Borrell, B. Echenard, D. Hitlin, D. Lin, S. Middleton, T. Miyashita, J. Oyang, F. Porter and R. Y. Zhu

California Institute of Technology, Pasadena, California, United States

P. Murat

Fermi National Accelerator Laboratory, Batavia, Illinois, United States

G. Pezzullo

Yale University, New Haven, Connecticut, United States

F. Grancagnolo, G. F. Tassielli

INFN - Sezione di Lecce, Lecce, Italy

\section{A. Zanetti \\ INFN - Sezione di Trieste, Trieste, Italy}

The Mu2e experiment at Fermi National Accelerator Laboratory will search for the CLFV neutrino-less conversion of a negative muon into an electron in the field of an aluminum nucleus. The Mu2e design has been studied to improve the single event sensitivity of four orders of magnitude with respect to the previous experiments and provide an indirect probe for New Physics energy scales up to thousands TeV. Mu2e is composed of a tracker, an electromagnetic calorimeter and an external veto for cosmic rays. The calorimeter provides a powerful particle identification and an online trigger, while operating in a harsh environment. We report on the status of the calorimeter components production, the quality assurance tests and the tests performed with a reduced-scale prototype.

40th International Conference on High Energy physics - ICHEP20

July 28 - August 6, 2020

Prague, Czech Republic (virtual meeting) 


\section{Introduction}

The Mu2e experiment at Fermilab [1] aims to search for the Charged Lepton Flavor Violating (CLFV) neutrino-less, coherent conversion of a negative muon into an electron in the field of an Aluminum nucleus. The experimental signature of the $\mu \rightarrow$ e conversion is a monoenergetic electron with an energy equal to the muon rest mass minus the corrections due to the nuclear recoil and the binding energy. For the Aluminum nucleus, the energy of the mono energetic electron is equal to $104.97 \mathrm{MeV}$. The layout of the Mu2e experiment at Fermilab is thoroughly described in [1]. A system of three superconductive solenoids is used to produce and transport a high intensity pulsed negative muon beam to the Aluminum target, where muons stop and form muonic atoms. The goal of the Mu2e detectors is detecting the conversion electron from the overwhelming background of particles originated from the standard physics processes which involve the muonic atoms.

\section{The Mu2e Calorimeter}

The Mu2e detector is composed of a straw-tube tracker and an electromagnetic calorimeter [2], located inside a large superconducting solenoid in a region with a quite uniform axial magnetic field of $1 \mathrm{~T}$. The main role of the calorimeter is to provide complementary information to the tracker and achieve a powerful $\mu / \mathrm{e}$ particle identification which is crucial in rejecting one of the most dangerous Mu2e backgrounds.

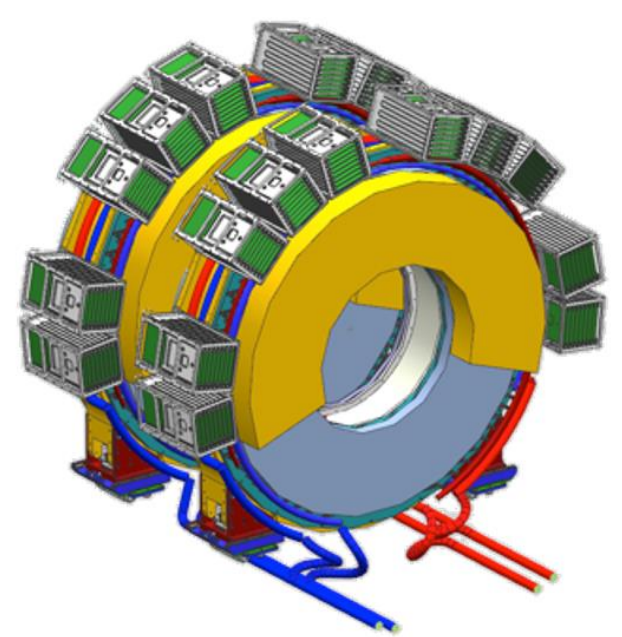

Figure 1: CAD drawings of the calorimeter disks. Layout of the digitization crates is also shown.

Thanks to a calorimeter-seeded track finder algorithm, it is also possible to increase the track reconstruction efficiency and make it more robust with respect to the occupancy level. The calorimeter information can also be used to implement a fast online trigger independent on the tracker. These tasks translate in the following requirements for $105 \mathrm{MeV}$ electrons: (a) a large acceptance; (b) a time resolution better than $500 \mathrm{ps;}$ (d) an energy resolution better than $10 \%$ and (d) a position resolution of the order of $1 \mathrm{~cm}$. The calorimeter should also be able to maintain a 
full functionality in a very harsh operation environment, inside a $10^{-4}$ Torr vacuum and $1 \mathrm{~T}$ magnetic field, and a very intense particles flux: depending on the position, a maximum of Total Ionizing Dose (TID) of the order of $100 \mathrm{krad} / \mathrm{year}$ and a neutron fluence of the order of $10^{12} \mathrm{n}_{\mathrm{eq}} / \mathrm{cm}^{2}$ are expected over five years of running. Since the cryostat will be accessible for maintenance on average only once per year, a high reliability is required for each calorimeter component. The presence of the magnetic field imposed the choice of Silicon PhotoMultipliers (SiPMs) as photosensors. The request on the light collection, obtained from the Monte Carlo simulation, is of at least 20 photo-electrons (p.e.)/MeV. To have a redundant system, each crystal is readout by two photosensors that must be able to independently collect the light. After a long R\&D program [3][4], the discussed requirements pushed the experiment to adopt a calorimeter made of undoped CsI crystals optically coupled to $14 \times 20 \mathrm{~mm}^{2}$ large area UV-extended SiPMs. The final calorimeter design consists of two matrices of 674 un-doped CsI crystals each coupled to two SiPMs and arranged in two disks as shown in Figure 1. Each disk has an internal (external) radius of $374 \mathrm{~mm}(660 \mathrm{~mm})$ and is filled with $34 \times 34 \times 200 \mathrm{~mm}^{3}$ crystals. The distance between the two disks is $75 \mathrm{~cm}$ to maximize the acceptance for the $105 \mathrm{MeV}$ signal electrons following a helical trajectory along the magnetic field.

\section{Undoped CsI Crystals}

Undoped CsI crystals were chosen as the best compromise between cost, performance and reliability: they offer a good radiation hardness, a fast decay time and a sufficient light yield; the main scintillation component is emitted at a wavelength of $310 \mathrm{~nm}$, well matching the Photon Detection Efficiency (PDE) of the new generation of UV-extended commercial SiPMs. To select the final producer for the crystals we organized an international bid involving three vendors: St. Gobain (France), SICCAS (China) and Amcrys (Ukraine). A total of 72 pre-production crystals of final dimension and shape were procured and exposed to a Quality Assurance process [5]. After the technical evaluation we decided to use both St. Gobain and SICCAS firms for the production of the calorimeter crystals, in order to minimize schedule risks.

\section{Silicon Photomultipliers}

To reach the requested light collection efficiency, a custom SiPM package has been developed: it consists of a $3 \times 2$ matrix of $6 \times 6 \mathrm{~mm}^{2}$ monolithic SiPMs (cells) with the readout organized as the parallel of 2 series of three cells [6]. With this configuration, the total capacitance is reduced to one third of the capacity of one single cell, so to have narrower signals and keep the pileup under control, at the cost of a triple bias voltage. After an accurate characterization of different models from different vendors [7], Hamamatsu devices (with 13360-6050CS used as cell) have been selected. These devices show a good PDE $(\sim 30 \%$ at $315 \mathrm{~nm})$ with a gain greater than $10^{6}$ at an over-voltage of $3 \mathrm{~V}$ with respect to the breakdown voltage. Following the calorimeter requirements, one important aspect to be considered is the radiation hardness: in this context, we have performed different tests with a ${ }^{60} \mathrm{Co}$ source up to the expected TID of $20 \mathrm{krad}$ (which do not show any significant effect on the leakage current) and with neutrons. When exposing sensors to $14 \mathrm{MeV}$ neutrons with a total flux of $2.2 \times 10^{11} \mathrm{n} / \mathrm{cm}^{2}$ (corresponding to 2.2 times the experiment lifetime), we have observed a too high increase of the leakage current (up to $2.3 \mathrm{~mA}$ when using $3 \times 3 \mathrm{~mm}^{2}$ Hamamatsu cells). To reduce the leakage current to an acceptable 
level, we need to cool down all SiPM to a temperature of $0{ }^{\circ} \mathrm{C}$. In order to do that, the calorimeter will exploit a dedicated cooling system.

\section{Electronics and Calibration}

The Front End Electronics (FEE) consists of two discrete and independent chips (Amp-HV), for each crystal, directly connected to the back of the SiPM. These provide the amplification and shaping stage, a local linear regulation of the bias voltage, monitoring of current and temperature on the sensors and a test pulse. Groups of 20 differential signals are sent to the waveform digitizer board called DIRAC. In order to limit the number of pass-through connectors, the DIRAC boards are hosted inside the cryostat which hosts the Mu2e detectors. All the components used for the FEE and the DIRAC [8] have been thoroughly tested and qualified for the harsh Mu2e radiation environment. The calibration of the whole calorimeter system will be done following several steps. The crystal by crystal equalization will be performed by means of a calibration system, where a $6.13 \mathrm{MeV}$ gamma line is obtained by irradiating a ${ }^{19} \mathrm{~F}$ fluid with low energy neutrons coming from a switchable external generator. The pipes with the ${ }^{19} \mathrm{~F}$ fluid are embedded in the calorimeter front plate to provide a uniform crystal irradiation. The source system is complemented with a laser system for the SiPMs and FEE calibration. The laser light is split using diffusive spheres and distributed to each crystal using optical fibers bundles.

\section{The Module-0}

In the year 2016 we assembled the first reduced-scale prototype of the calorimeter, the so called 102 SiPMs handled by their FEE chips, with the mechanics and the cooling system as similar as possible to the final ones. This prototype had a key role in validating the expected physics performance and also checking several mechanical properties, such as the performance of the cooling system and the assembly procedures. During May 2017 a test beam was performed at the Beam Test Facility in Frascati (Italy) using an electron beam in the range 60 to $120 \mathrm{MeV}$. The measured performance [9] for particles impinging on the calorimeter surface with an impact angle of $0 \circ$ and $50^{\circ}$ (the average angle expected from conversion electrons) is shown in the plots of Figure 2: an energy resolution of about 5\% (7\%) and a time resolution of about $120 \mathrm{ps}$ (150 ps) have been obtained for $100 \mathrm{MeV}$ electrons at $0^{\circ}\left(50^{\circ}\right)$. A good agreement between test beam data and Monte Carlo simulation is also evident. These results widely satisfied the Mu2e experimental requirements and the technological choices made for the Module- 0 were approved for the construction of the Mu2e calorimeter.

\section{Status of the production}

Starting from March 2018, we received one batch of 300 photosensors/month and 60 crystals/month at Fermilab. The Quality Assurance (QA) process has been carried out in a dedicated soft clean room at the SiDet Fermilab department [10,11]. For each crystal we measured the geometrical accuracy (100 $\mu \mathrm{m}$ of tolerance) on both dimensions and shape using the SiDET 

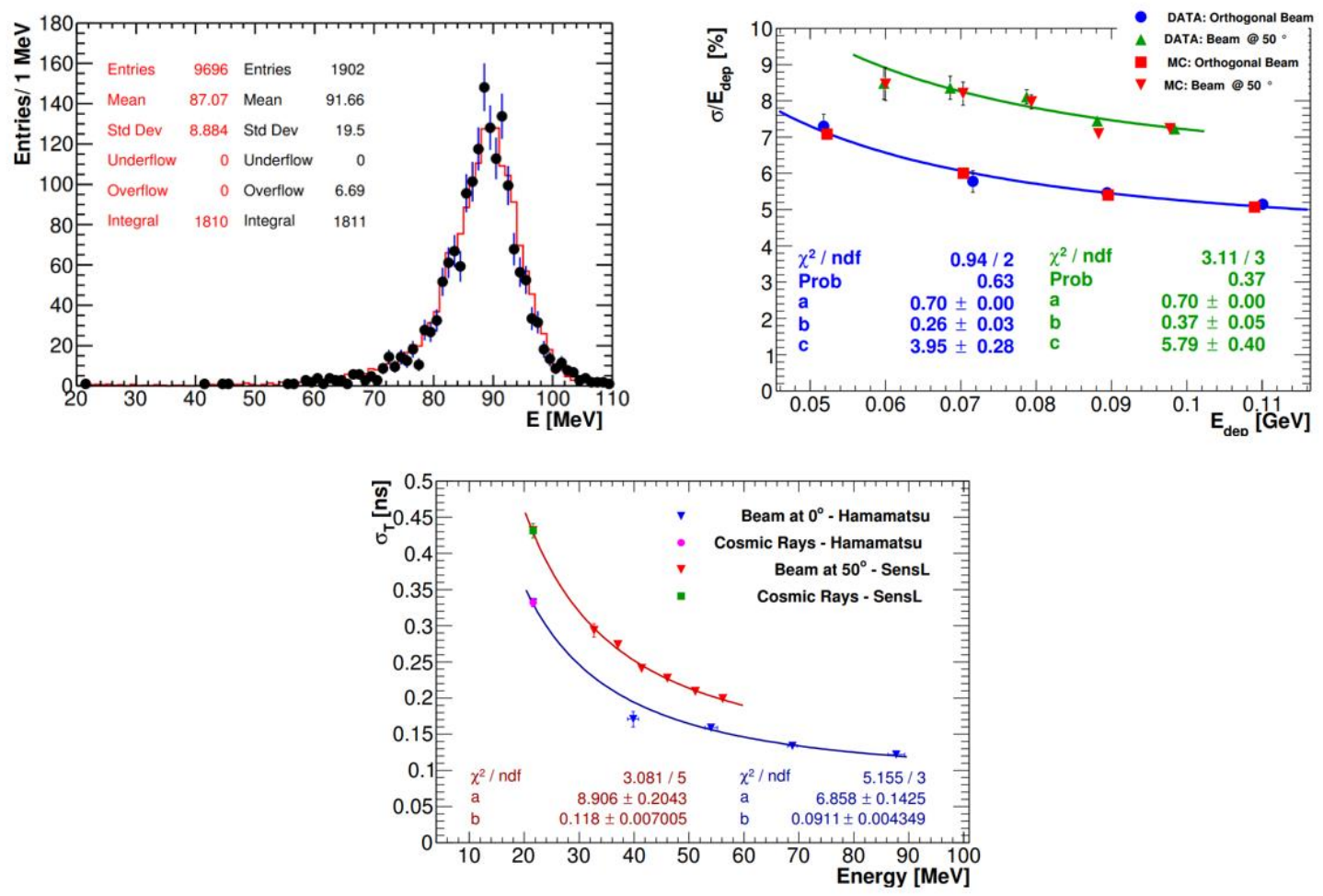

Figure 2: Calorimeter performance evaluated with the Module-0, using 60-120 MeV electron beam. On the left plot is shown the data-MC comparison of the deposited energy distribution for $100 \mathrm{MeV}$ electrons. On the right (bottom) plot is instead shown the energy (time) resolution for orthogonal and 50 - impinging electrons as a function of the deposited energy in the Module- 0 crystals (the most energetic crystal). The beam energy steps are of $20 \mathrm{MeV}$ with orthogonal beam and of $10 \mathrm{MeV}$ with tilted beam.

Coordinate Measuring Machine and the optical quality. The parameters used to evaluate the optical quality are the light yield, the Longitudinal Response Uniformity (LRU), the energy resolution at $511 \mathrm{keV}$ and the Fast/Total component (i.e., the ratio of the energy measured integrating the signal in $200 \mathrm{~ns}$ with respect to the total signal integrated in $3 \mu \mathrm{s}$ ). About $10 \%$ of the crystals have been rejected, mostly due to problems with mechanical tolerance. SiPMs selection criteria required a good uniformity among the cells of the same device and a PDE that guarantees the requested light collection of 20 photoelectrons/MeV. The breakdown voltage, the gain and the dark current were measured for each of the six cells of each device at three temperatures $\left(-10^{\circ} \mathrm{C}, 0^{\circ} \mathrm{C}\right.$ and $\left.20^{\circ} \mathrm{C}\right)$ under a $10^{-4}$ Torr vacuum. The overall rejection factor was at the level of $1.2 \%$ and was dominated by sensors with a high dark current RMS. The irradiation tests were carried out on small crystals and SiPM subsamples from each production batch: no variations were observed in the occurred radiation damage over the monitored phases of the production.

\section{Conclusions}

The characterization of the active components purchased for the construction of the Mu2e electromagnetic calorimeter confirmed the excellent quality of the selected producers. Detector 
construction is in progress and is expected to be completed by the end of 2021. Installation will take place in 2022, followed by a commissioning phase in 2022-2023.

\section{Acknowledgements}

We are grateful for the vital contributions of the Fermilab staff and the technical staff of the participating institutions. This work was supported by the US Department of Energy; the Istituto Nazionale di Fisica Nucleare, Italy; the Science and Technology Facilities Council, UK; the Ministry of Education and Science, Russian Federation; the National Science Foundation, USA; the Thousand Talents Plan, China; the Helmholtz Association, Germany; and the EU Horizon 2020 Research and Innovation Program under the Marie Sklodowska-Curie Grant Agreement No. $690835,734303,822185,858199$. This document was prepared by members of the Mu2e Collaboration using the resources of the Fermi National Accelerator Laboratory (Fermilab), a U.S. Department of Energy, Office of Science, HEP User Facility. Fermilab is managed by Fermi Research Alliance, LLC (FRA), acting under Contract No. DE-AC02-07CH11359.

\section{References}

[1] L. Bartoszek, et al., Mu2e technical design report (2014).

[2] N. Atanov, et al., The calorimeter of the Mu2e experiment at Fermilab, JINST 12 (01) (2017) C01061.

[3] N. Atanov et al., Measurement of time resolution of the Mu2e LYSO calorimeter prototype, Nucl.Instrum.Meth. A812 (2016) 104-111.

[4] O. Atanova et al., Measurement of the energy and time resolution of a undoped CsI + MPPC array for the Mu2e experiment, JINST 12 (2017) no.05, P05007.

[5] N. Atanov et al., Quality Assurance on Undoped CsI Crystals for the Mu2e Experiment, IEEE Trans.Nucl.Sci. 65 (2017) no.2, 752-757.

[6] I. Sarra, et al., The Mu2e Calorimeter Photosensors, Springer Proc. Phys. 213 (2018) 275-279.

[7] G Cordelli, M., et al., Pre-production and quality assurance of the Mu2e calorimeter Silicon Photomultipliers, Nucl.Instrum.Meth. A912 (2018) 347-349.

[8] S. Di Falco et al., Components Qualification for a Possible use in the Mu2e Calorimeter Waveform Digitizer, JINST 12 (2017) no.03, C03088.

[9] N. Atanov et al., Electron beam test of the large area Mu2e calorimeter prototype, J.Phys.Conf.Ser. 1162 (2019) no.1, 012027.

[10] D. Caiulo et al., Production and Quality Assurance of the Mu2e Calorimeter Silicon Photomultipliers, J.Phys.Conf.Ser. 1162 (2019) no.1, 012024.

[11] N. Atanov et al., The Mu2e Calorimeter: Quality Assurance of Production Crystals and SiPMs, Nucl.Instrum.Meth. A936 (2019) 154-155. 